\title{
Electroacupuncture potentiates peripheral CB2 receptor-inhibited chronic pain in a mouse model of knee osteoarthritis
}

This article was published in the following Dove Press journal: Journal of Pain Research

\author{
Xiao-Cui Yuan,' Qiang \\ Wang, ${ }^{2}$ Wen Su, ${ }^{3}$ Hong- \\ Ping Li,' Cai-Hua Wu, ${ }^{3}$ Fang \\ Gao,' Hong-Chun Xiang,' \\ He Zhu,' Li-Xue Lin,' Xue- \\ Fei $\mathrm{Hu},{ }^{\prime}$ Jie $\mathrm{Cao},{ }^{4}$ Jing-Jing \\ Li,' Man Li' \\ 'Department of Neurobiology and \\ Key Laboratory of Neurological \\ Diseases of Ministry of Education, The \\ Institute of Brain Research, School \\ of Basic Medicine, Tongji Medical \\ College of Huazhong University \\ of Science and Technology, Wuhan \\ 430030, People's Republic of China; \\ ${ }^{2}$ Department of Anesthesiology, First \\ Affiliated Hospital of Xi'an JiaoTong \\ University, Xi'an, People's Republic of \\ China; ${ }^{3}$ Department of Acupuncture, \\ Wuhan First Hospital, Wuhan \\ 430030, People's Republic of China; \\ ${ }^{4}$ Department of Neurology, Tongji \\ Hospital, Tongji Medical College of \\ Huazhong University of Science and \\ Technology, Wuhan 430030, People's \\ Republic of China
}

Correspondence: Man Li; Jing-Jing Li Department of Neurobiology, Tongji Medical College of Huazhong University of Science and Technology, 13 Hangkong Road, Wuhan 430030, Hubei, People's

Republic of China

Tel +862783692630

Fax +862783692608

Email liman73@mails.tjmu.edu.cn;

jingjing-lee@foxmail.com
Purpose: Knee osteoarthritis (KOA) is a highly prevalent, chronic joint disorder, with chronic pain as its typical symptom. Although studies have shown that an activated peripheral CB2 receptor can reduce acute pain, whether the CB2 receptor is involved in electroacupuncture (EA) inhibiting chronic pain and the involved mechanism remains unclear. The aim of this study was to investigate whether EA may strengthen peripheral CB2 receptor-inhibited chronic pain in a mouse model of KOA.

Materials and methods: KOA was induced by intra-articular injection of monosodium iodoacetate (MIA) into the left knee joint of mice. Thermal hyperalgesia was tested with the hot plate test, and mechanical allodynia was quantified using von Frey filaments. The expression of CB2 receptor and IL-1 $\beta$ were quantified by using immunofluorescence labeling.

Results: EA treatment at $2 \mathrm{~Hz}+1 \mathrm{~mA}$ significantly increased the expression of CB2 receptor in fibroblasts and decreased the expression of IL-1 $\beta$ in the menisci compared with that in the KOA group. However, EA had no effect on the expression of IL- $1 \beta$ in CB2 $2^{-/}$mice. At $2 \mathrm{~Hz}+1 \mathrm{~mA}$, EA significantly increased mechanical threshold, thermal latency, and weight borne after KOA modeling. However, knockout of the CB2 receptor blocked these effects of EA. After $2 \mathrm{~Hz}+1 \mathrm{~mA}$ treatment, EA significantly reduced the Osteoarthritis Research Society International (OARSI) score after KOA modeling. However, EA had no significant effect on the OARSI score in $\mathrm{CB} 2^{-/}$mice.

Conclusion: EA reduced the expression of IL-1 $\beta$ by activating the $\mathrm{CB} 2$ receptor, thus inhibiting the chronic pain in the mouse model of KOA.

Keywords: cannabinoid, acupuncture, inflammatory pain, IL-1 $\beta$

\section{Introduction}

Chronic pain is often persistent and poorly treated by existing therapies in clinic, which is an important focus of research on the mechanism of pain and acupuncture analgesia. ${ }^{1,2}$ It has been proved that electroacupuncture (EA) is effective in relieving chronic pain in patients with knee osteoarthritis (KOA). ${ }^{3}$ However, the involved mechanisms remain unclear.

The endocannabinoid (EC) system consists of the following two main receptors: CB1 receptor, which is localized primarily on the central nervous system and peripheral neurons, ${ }^{4,5}$ and CB2 receptor, which is found principally in the immune system and to a lesser extent in the central nervous system. ${ }^{6-9}$ In our previous studies, we found that endogenous cannabinoids and peripheral CB2 receptor are involved in the antinociceptive effect of EA on acute inflammatory pain. ${ }^{10-14}$ Moreover, other studies 
also found that activated peripheral CB2 receptor reduces inflammatory pain and neuropathic pain. ${ }^{15,16} \mathrm{KOA}$ is a highly prevalent, chronic joint disorder, with chronic pain as its typical symptom. ${ }^{17,18}$ Furthermore, the overexpression of CB2 receptor is associated with a reduced pain phenotype in monosodium iodoacetate (MIA)-treated mice. ${ }^{19}$ Thus, we wondered whether EA may inhibit chronic pain via the CB2 receptor in a mouse model of KOA.

Oversecretion of pro-inflammatory cytokines, including TNF- $\alpha$, IL- 6 , and IL- $1 \beta$, contributes to the severity and the progression of osteoarthritis. ${ }^{20}$ High levels of pro-inflammatory cytokines in bones and joints induce pain, cartilage loss, and even joint dysfunction. ${ }^{20,21}$ Furthermore, we have shown that EA inhibited the release of inflammatory cytokines IL-1 $\beta$, IL-6, and TNF-a through the CB2 receptor in inflammatory skin tissues, thus attenuating acute inflammatory pain. ${ }^{11}$ Therefore, reducing the release of pro-inflammatory cytokines is an effective therapy for KOA.

Therefore, in this study, we first determined the level of CB2 receptor and inflammatory cytokine IL-1 $\beta$. There was no significant difference in pain behavior, Osteoarthritis Research Society International (OARSI) scores, and body weight between the $\mathrm{CB} 2$ receptor knockout mice $\left(\mathrm{CB} 2^{-/-}\right.$ mice) and wild-type mice in different KOA models or was there any obvious difference between genotypes in general health status, behavior, or locomotor activity. ${ }^{19,22}$ So, we used the $\mathrm{CB} 2^{-/-}$mice to determine whether the $\mathrm{CB} 2$ receptor was involved in the effects of EA inhibiting the chronic pain in the model of KOA.

\section{Materials and methods Animals}

All animal experiments were approved by the Animal Care and Use Committee at the Huazhong University of Science and Technology and conformed to the ethical guidelines of the International Association for the Study of Pain. ${ }^{23}$ Eight-week-old female C57BL/6 mice were obtained from the Experimental Animal Center of Tongji Medical College of Huazhong University of Science and Technology. CB2 receptor knockout mice $\left(\mathrm{CB} 2^{-/-}\right.$mice) maintained on a C57BL/6 congenic background were kindly provided by $\mathrm{Dr}$ Nancy E Buckley (Basic Neuroscience Program, NINDS, National Institutes of Health, Bethesda, MD, USA). Female $\mathrm{CB}^{-/-}$mice and their wild-type littermate $\left(\mathrm{CB} 2^{+/+}\right)$mice were generated as described previously. ${ }^{24}$ The mice were individually housed in cages with a 12-hour light/dark cycle and had free access to food and water.
In our previous study, we have demonstrated that the optimized parameters of EA inhibiting chronic pain were the low frequency and high intensity $(2 \mathrm{~Hz}+1 \mathrm{~mA})$ and that the sham EA group had no significant effect on pain behavior of KOA mice. ${ }^{25}$ Therefore, in this study, the following three groups of mice were used: control, KOA, and $2 \mathrm{~Hz}+1 \mathrm{~mA} \mathrm{EA}$.

\section{Induction of KOA}

KOA was induced by intra-articular injection of MIA (SigmaAldrich Co., St Louis, MO, USA) into the left knee joint after mice were briefly anesthetized with $10 \%$ chloralic hydras. The knee joint was shaved and flexed at a $90^{\circ}$ angle. $5 \mu \mathrm{L}$ of $5 \mathrm{mg} / \mathrm{mL}$ MIA in sterile saline $(0.9 \%)$ was injected through the infrapatellar ligament into the joint space of the left knee with a $30 \mathrm{G}$ needle. ${ }^{19}$ This concentration of MIA causes histological changes in the cartilage ${ }^{26}$ and induces joint pain ${ }^{27}$ in mice. The control mice received an intra-articular injection of vehicle ( $5 \mu \mathrm{L}$ of sterile saline, $0.9 \%$ ).

\section{EA treatment}

The animals were habituated to the restricting bag for 3 days before KOA induction, 30 minutes each day. In the EA treatment group, mice received EA administration on the left "Neixiyan" (Ex-LE4) and "Dubi" (ST35) once every other day for 4 weeks, starting from 2 days after MIA injection. EA ( $1 \mathrm{~mA}$ and $0.1 \mathrm{~ms}$ ) was administered at the $2 \mathrm{~Hz}$ frequency for 30 minutes. The EA treatment and behavioral analysis are not carried out on the same day. The mice received EA administration in the even-numbered days after MIA injection, and behavioral analysis was done in the odd-numbered days after MIA injection. Current was delivered with a Han's Acupoint Nerve Stimulator (LH202; Huawei Co., Ltd., Beijing, China).

Two acupuncture needles were inserted into two acupoints corresponding to Ex-LE4 and ST35 in humans. Ex-LE4 is located at the medial cavity of the patella and the patellar ligament, and ST35 lies on the lateral cavity of the patella and patellar ligament. Ex-LE4 and ST35 were chosen, because they are frequently used in KOA and are specific acupoints for treating knee problems. ${ }^{28,29}$

\section{Nociceptive behavioral tests}

Mechanical allodynia and heat hyperalgesia were also demonstrated in the hind paw of animals with KOA, using von Frey filaments and the hot plate test, respectively. ${ }^{23,30}$ Animals were first allowed to acclimate to the testing apparatus for 30 minutes a day, 3 days before MIA injection. The baseline nociceptive thresholds were tested for 3 days before MIA 
injection, and the mean value was calculated as baseline. The nociceptive thresholds were tested once every other day, starting from the first day after KOA induction for 4 weeks.

The hot plate test was used to measure the response latencies according to a previously described method. ${ }^{31} \mathrm{~A}$ glass cylinder ( $40 \mathrm{~cm}$ high, $20 \mathrm{~cm}$ diameter) was used to keep mice on the hot surface of the plate, which was maintained at a temperature of $55^{\circ} \mathrm{C} \pm 0.5^{\circ} \mathrm{C}$. The time between placement of the mouse and the shaking or licking of paws or jumping was recorded as the index of response latency. A latency period (cutoff) of 30 seconds was defined as complete analgesia. ${ }^{32}$ The test was repeated three times in mice, and the mean value was calculated.

Mechanical allodynia was assessed by placing mice on an elevated mesh floor, and the tactile threshold was measured by using the "up-down" method. ${ }^{33}$ After an acclimation period of 30 minutes, a series of calibrated von Frey filaments (Stoelting, Wood Dale, IL, USA) were applied perpendicularly to the plantar surface of the left hind paw with sufficient force to bend the filament for 6 seconds. Brief withdrawal or paw flinching was considered as a positive response. The test was repeated two times in mice, and the mean value was calculated.

\section{Weight-bearing assessment}

The spontaneous joint pain behavior was assessed using a hind limb weight-bearing apparatus (Model-600M/R; IITC Life Science, Woodland Hills, CA, USA). Mice were allowed to acclimate to the testing apparatus for 30 minutes a day, 3 days before MIA injection. The amount of weight supported by each hind leg was measured automatically. The test was repeated five times in mice, and the mean value was calculated. Weight-bearing difference between the two legs was presented as the percentage of weight borne by the left leg and was determined using the following formula: \% weight on the left leg = weight on the left leg/(weight on the left leg+weight on the right leg) $\times 100 \%$. $^{34}$

\section{Safranin O-Fast Green staining}

Four weeks after the experimental induction of KOA, mice were deeply anesthetized with $10 \%$ chloralic hydras and intracardially perfused with $37^{\circ} \mathrm{C}$ normal saline followed by $4 \%$ paraformaldehyde in $0.1 \mathrm{M}$ phosphate buffer $(\mathrm{pH}$ $7.4 ; 4^{\circ} \mathrm{C}$ ). The left knee joints were subsequently removed, post-fixed in $4 \%$ paraformaldehyde for 24 hours, and then the knee joints were decalcified in 10\% EDTA for 2 weeks on a plate shaker at room temperature (RT). ${ }^{35}$ The decalcified knee joints were washed in $0.1 \mathrm{M}$ phosphate buffer $(\mathrm{pH}$ 7.4) overnight. After dehydrating the tissues with increasing concentrations of ethanol and xylene transparent, the joints were subsequently embedded in wax. Coronal $4 \mu \mathrm{m}$ sections were cut in a pathological slicer from the frontal plane toward the back of each joint and mounted on gelatinized slides.

All the serial sections were stained with the Safranin O-Fast Green staining protocol. Briefly, after hydrating sections with decreasing concentrations of ethanol, the sections were stained with $1 \%$ Safranin $\mathrm{O}$ and subsequently with $0.5 \%$ Fast Green solutions. Finally, the sections were dehydrated and cleared with increasing ethanol concentrations and xylene, and then mounted with rhamsan gum and a covering glass. All the stained sections were viewed at $10 \times$ objective with an Olympus BX51 fluorescence microscope (Olympus Corporation, Tokyo, Japan). Images were captured using a Qimaging Micropublisher RTV 5.0 microscope camera and QCapture Pro 6.0 software (Qimaging, Surrey, BC, Canada). A total of five to six slides were randomly selected in each knee joint. All images of the obtained sections spanning the central loadbearing region of the knee were taken for both medial and lateral sides of each joint and used for histological scoring.

Histological evaluation of the severity of KOA was performed by an observer (XCY) blinded to genotype and pharmacological treatment according to the OARSI scoring system. ${ }^{35}$ All the following four quadrants of the knee joint were evaluated: medial tibial plateau (MTP), medial femoral condyle (MFC), lateral tibial plateau (LTP), and lateral femoral condyle (LFC). The scores were expressed as the summed histological score. The summed score represents the additive scores for each quadrant of the joint on each section through the joint of each animal. Then, the average summed score for each experimental group was calculated.

A randomly selected sample of 12 joints was assessed by a second observer (FG), who was also blinded to genotype and treatment allocation to test reproducibility of the scoring system.

\section{Immunofluorescence labeling}

The knee joints were decalcified as described previously. ${ }^{35}$ The decalcified knee joints were cryoprotected in $30 \%$ sucrose in $0.1 \mathrm{M}$ phosphate buffer for 24 hours at $4^{\circ} \mathrm{C}$. The sections were cut at $20 \mu \mathrm{m}$ on a cryostat, which were mounted onto gelatin-coated slides and dried overnight.

The sections were rinsed in 0.01 M PBS, blocked for 1 hour with 5\% donkey serum and $0.2 \%$ Tween-20 in PBS and then incubated with the following primary antibodies at $37^{\circ} \mathrm{C}$ for 1 hour and at $4^{\circ} \mathrm{C}$ overnight: rabbit anti-CB2 (1:100; Abcam, Hong Kong, China); mouse anti-S100A4 (1:100; Abcam); and rabbit anti-IL-1 $\beta$ (1:50; Santa Cruz 
Biotechnology Inc., Dallas, TX, USA). Subsequently, the sections were washed four times in PBS for 5 minutes and incubated with the corresponding secondary antibodies from Jackson ImmunoResearch Laboratories, Inc. (West Grove, PA, USA): donkey anti-rabbit IgG conjugated with Dylight 488 (1:400) and donkey anti-mouse IgG conjugated with Dylight 594 (1:400). The sections were washed four times in PBS for 5 minutes and then incubated with DAPI (1:1,000; Abcam). The sections were washed four times in PBS for 5 minutes and then coverslipped. Negative controls were included by omitting the primary antibodies and with primary antibodies preabsorbed with their specific blocking peptides in the abovementioned procedures, which resulted in no positive labeling in the knee joint tissues. For quantification, a total of 5-6 sections from the knee joint were randomly selected for each mouse. Digital images were acquired using an Olympus BX51 fluorescence microscope (Olympus Corporation). Images were captured using a Qimaging Micropublisher RTV 5.0 microscope camera and QCapture Pro 6.0 software (Qimaging). All images for each experiment were taken at the same time with the same camera settings, and the authors performing the image analysis were blinded to the group. The number of CB2 with DAPI or S100A4positive cells and the area of IL- $1 \beta$ in the menisci were measured by using Image $\mathrm{J}$ software.

\section{Statistical analyses}

Using pilot data, we estimated the sample size based on at least $80 \%$ power and $\alpha=0.05$. Data are presented as mean \pm SD. To determine the statistical difference in the withdrawal thresholds between different groups and time points, we used two-way repeated ANOVA followed by Bonferroni's post hoc test. One-way ANOVA and Newman-Keuls post hoc test were used in biochemical data. A $P$-value of less than 0.05 was considered statistically significant.

\section{Results}

EA reverses the reduction of $\mathrm{CB} 2$ receptor and the increase in IL-I $\beta$ expression in the menisci of KOA mice

The CB2 receptor-positive cells were present in the menisci (Figure 1A). The number of CB2 receptor-positive cells in the menisci was significantly lower in the KOA group than the control group (Figure 1B). EA treatment at $2 \mathrm{~Hz}+1 \mathrm{~mA}$ significantly increased the number of $\mathrm{CB} 2$-positive cells in the menisci compared with that in the KOA group (Figure 1B).
The area of IL-1 $\beta$-positive cells in the menisci was significantly increased in the KOA group than the control group (Figure $1 \mathrm{C}$ ). EA treatment at $2 \mathrm{~Hz}+1 \mathrm{~mA}$ significantly decreased the area of IL-1 $\beta$-positive cells in the menisci compared with that in the KOA group (Figure 1C).

\section{EA reverses the reduction of CB2 receptor expression in fibroblasts of KOA mice}

Previous studies have proved that the expression of CB2 was demonstrated in synovial fibroblasts (SF). ${ }^{36,37}$ To dissect the role of $\mathrm{CB} 2$ receptor in fibroblasts, we investigated the colocalization between CB2 and the fibroblast marker S100A4 immunoreactivities.

The CB2 and S100A4-positive cells were present in the menisci (Figure 2A). Compared with the control group, MIA significantly decreased the percentage of double-stained CB2 and S100A4 in the menisci (Figure 2B). EA treatment significantly increased the percentage of double-stained CB2 and S100A4 in the menisci (Figure 2B). In addition, the number of S100A4-positive cells in the menisci did not differ significantly among different groups (Figure 2C).

\section{CB2 receptor is involved in the EA effects on pain hypersensitivity in KOA mice}

In the wild-type mice, KOA induction significantly reduced mechanical withdrawal threshold and thermal withdrawal latency (Figure 3A and C). At $2 \mathrm{~Hz}+1 \mathrm{~mA}$, EA significantly increased tactile threshold and thermal latency after KOA modeling (Figure $3 \mathrm{~A}$ and $\mathrm{C}$ ). In $\mathrm{CB} 2^{-/-}$mice, KOA modeling still decreased tactile threshold and thermal latency. However, EA had no significant effects on mechanical threshold and thermal latency after KOA (Figure 3B and D).

Before MIA was injected into the knee joint cavity, there was no significant difference between right and left hind limb weight bearing in the groups (Figure 3E and F). In the wild-type mice, KOA induction significantly reduced the percentage of weight borne (Figure $3 \mathrm{E}$ ). At $2 \mathrm{~Hz}+1 \mathrm{~mA}$, EA significantly increased the percentage of weight borne in KOA mice (Figure $3 \mathrm{E}$ ). In $\mathrm{CB}^{-/-}$mice, KOA modeling also decreased the percentage of weight borne. However, EA had no significant effect on the percentage of weight borne after KOA (Figure 3F). It suggested that the $\mathrm{CB} 2$ receptor participates in EA-inhibited chronic pain in KOA mice. 


\section{A \\ ญิ} CON

KOA EA
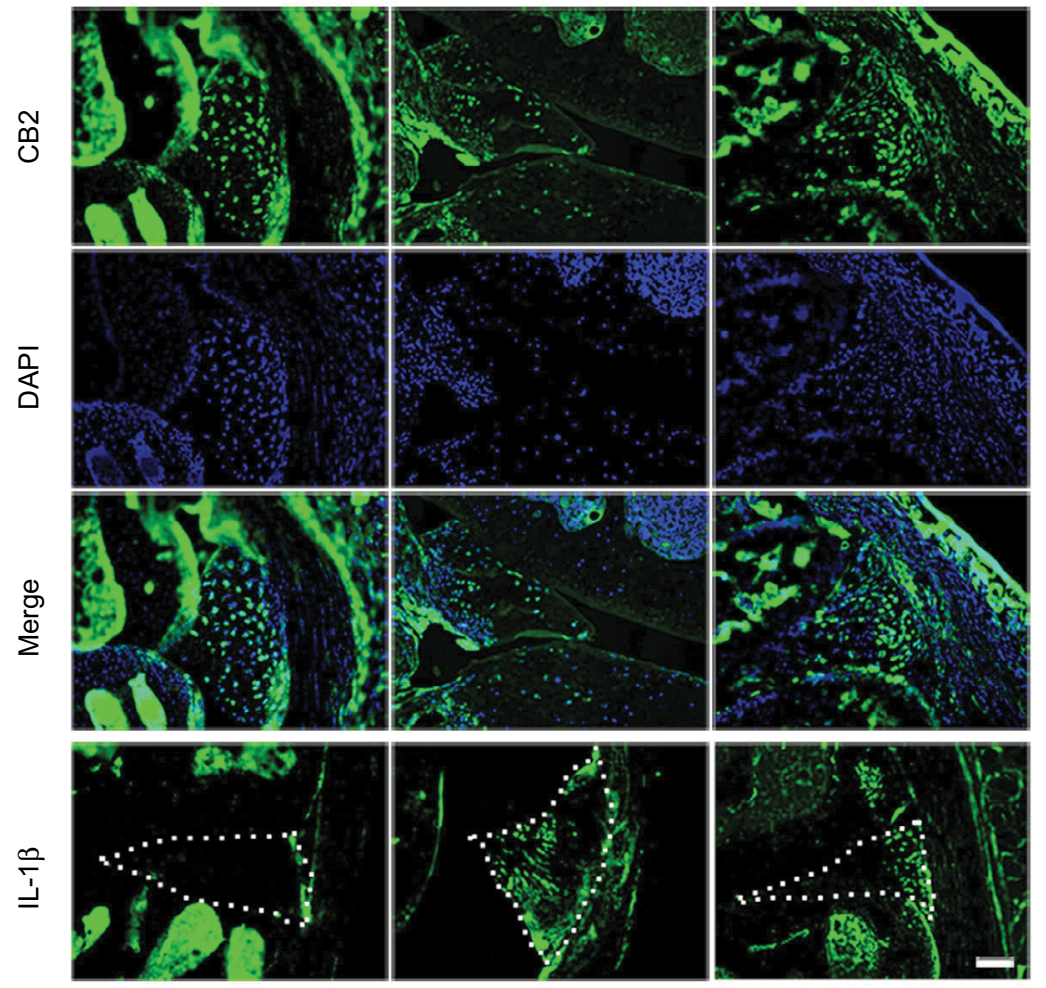

B
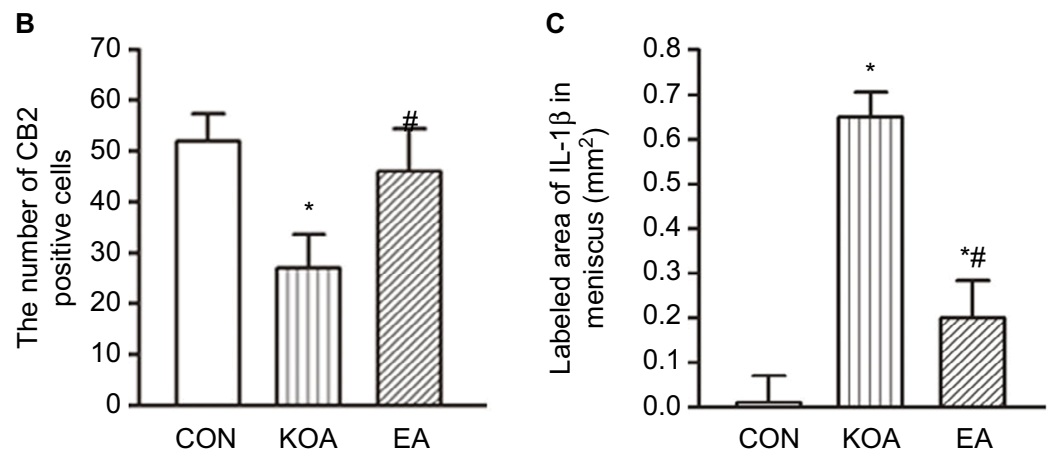

Figure I Quantitative analysis of the expression of CB2 receptor and IL-I $\beta$ in the menisci.

Notes: (A) Representative images showing CB2-immunoreactive cells, DAPI, and IL-I $\beta$ in the menisci of CON, KOA, and EA groups. Scale bar, $100 \mu \mathrm{m}$. Dotted line marks the menisci. (B) Summary data show the number of CB2-immunoreactive cells in different groups. (C) Summary data show the area of IL-I $\beta$ in different groups. Data are expressed as mean $\pm S D$ ( $n=6$ mice in each group). ${ }^{*}<<0.05$, compared with the control group; ${ }^{*} P<0.05$, compared with the KOA group.

Abbreviations: CON, control; EA, electroacupuncture; KOA, knee osteoarthritis.

CB2 receptor is involved in the improving effect of EA on histopathological

\section{alterations in KOA mice}

The OARSI scoring system is used for evaluating cartilage alterations. The primary slides were stained for cartilage proteoglycan using a Safranin O-Fast Green technique, which can be utilized with the scoring paradigm. ${ }^{19,35}$ Reproducibility of the OARSI scoring system used to assess severity of KOA was independently assessed by two observers (XCY and FG) in 12 out of 36 samples $(33.3 \%)$. This showed an inter-observer kappa co-efficient of $0.786(P<0.001)$, which is considered substantial. ${ }^{38}$

Representative images from serial histological sections of the wild-type and $\mathrm{CB} 2^{-/-}$mice stained with Safranin O-Fast Green are presented in Figure 4A. There was no significant difference in OARSI scores for the saline injection joints between the wild-type and $\mathrm{CB}^{-/-}$mice (Figure S1). The OARSI score for cartilage damage indicated that the saline injection did not 

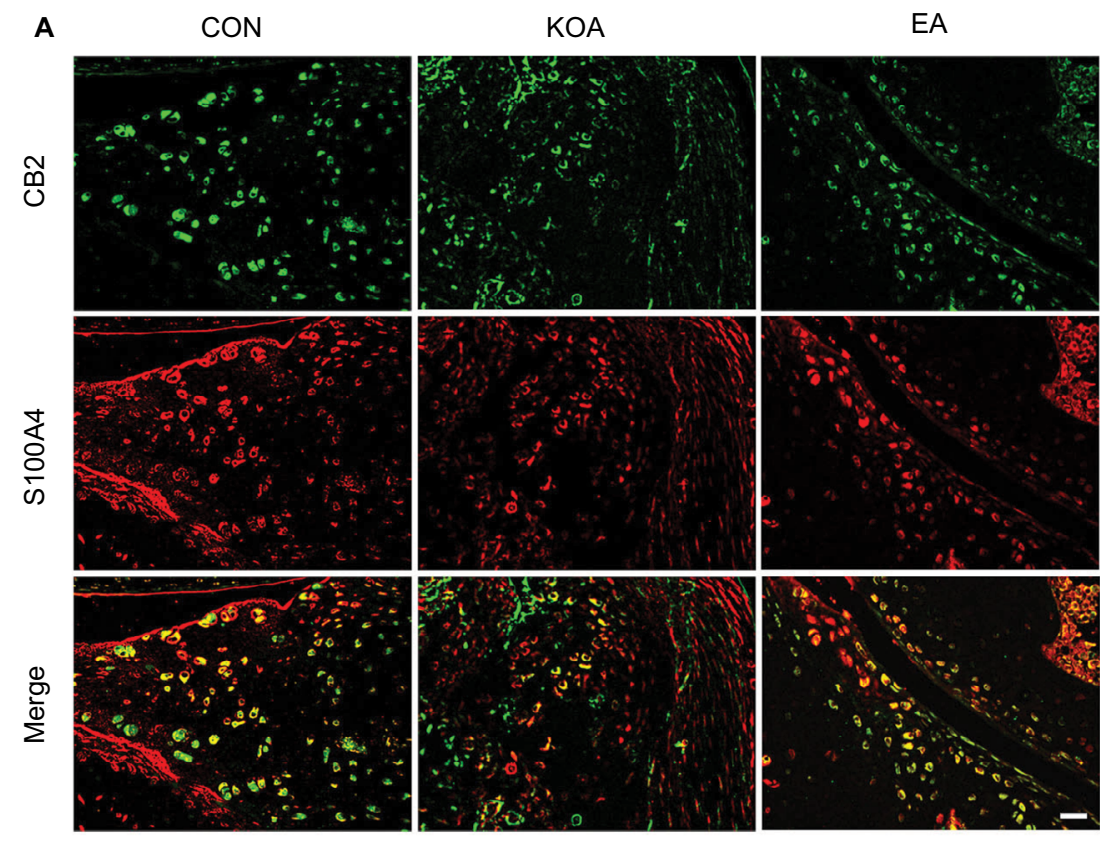

B

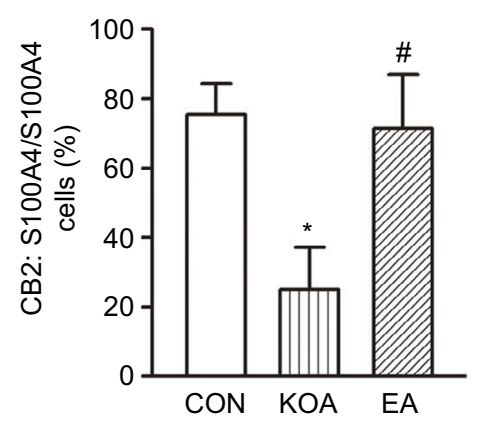

C

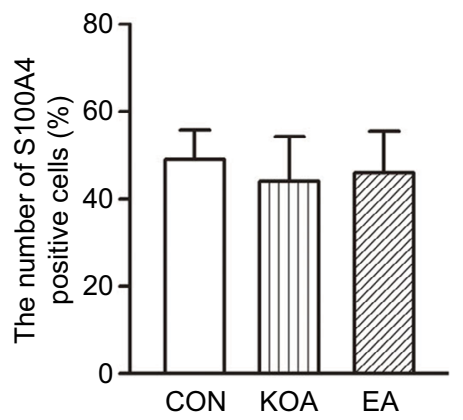

Figure 2 Double immunolabeling of CB2 and the fibroblast marker S100A4 in the menisci.

Notes: (A) CB2 labeling (green); SI00A4 labeling (red); and double-labeled cells (yellow). Scale bar, $20 \mu \mathrm{m}$. (B) Summary data show the percentage of double-stained cells $\%$ in the number of total SI00A4-immunoreactive cells. (C) Summary data show the number of SI00A4-immunoreactive cells in different groups. Data are expressed as mean $\pm S D$ ( $n=6$ mice in each group). ${ }^{*} P<0.05$, compared with the control group; ${ }^{*} P<0.05$, compared with the KOA group.

Abbreviations: CON, control; EA, electroacupuncture; KOA, knee osteoarthritis.

induce histological alterations in the knee joint of the wild-type and $\mathrm{CB} 2^{-/-}$mice (Figure $4 \mathrm{~B}$ and $\mathrm{C}$ ). In contrast, the intraarticular injection of MIA induced significant increase in the OARSI score in the knee joint of the wild-type and $\mathrm{CB}^{-/-}$mice (Figure 4B and C). Therefore, after intra-articular injection of MIA, the $\mathrm{CB} 2^{-/}$mice developed similar histological alterations to the wild-type mice in the knee joint.

After $2 \mathrm{~Hz}+1 \mathrm{~mA}$ treatment, EA significantly reduced the OARSI score after KOA modeling in wild-type mice (Figure 4B). However, EA had no significant effect on the OARSI score after KOA modeling in $\mathrm{CB} 2^{-/-}$mice (Figure 4C). It suggested that the $\mathrm{CB} 2$ receptor mediated the EA improvement of the histopathological alterations.
CB2 receptor is involved in the EA effect on reduction of the level of IL-I $\beta$ expression in the menisci of KOA mice

In the wild-type mice, the area of IL-1 $\beta$-positive cells in the menisci was significantly increased than that in the control group 4 weeks after KOA induction (Figure 5A and B). At $2 \mathrm{~Hz}+1 \mathrm{~mA}$, EA significantly reduced the area of IL-1 $\beta$-positive cells after KOA modeling (Figure $5 \mathrm{~A}$ and $\mathrm{B})$. In $\mathrm{CB} 2^{--}$mice, KOA modeling increased the area of IL-1 $\beta$-positive cells. However, EA had no significant effect on the area of IL-1 $\beta$-positive cells after KOA modeling (Figure $5 \mathrm{~A}$ and $\mathrm{C}$ ). 

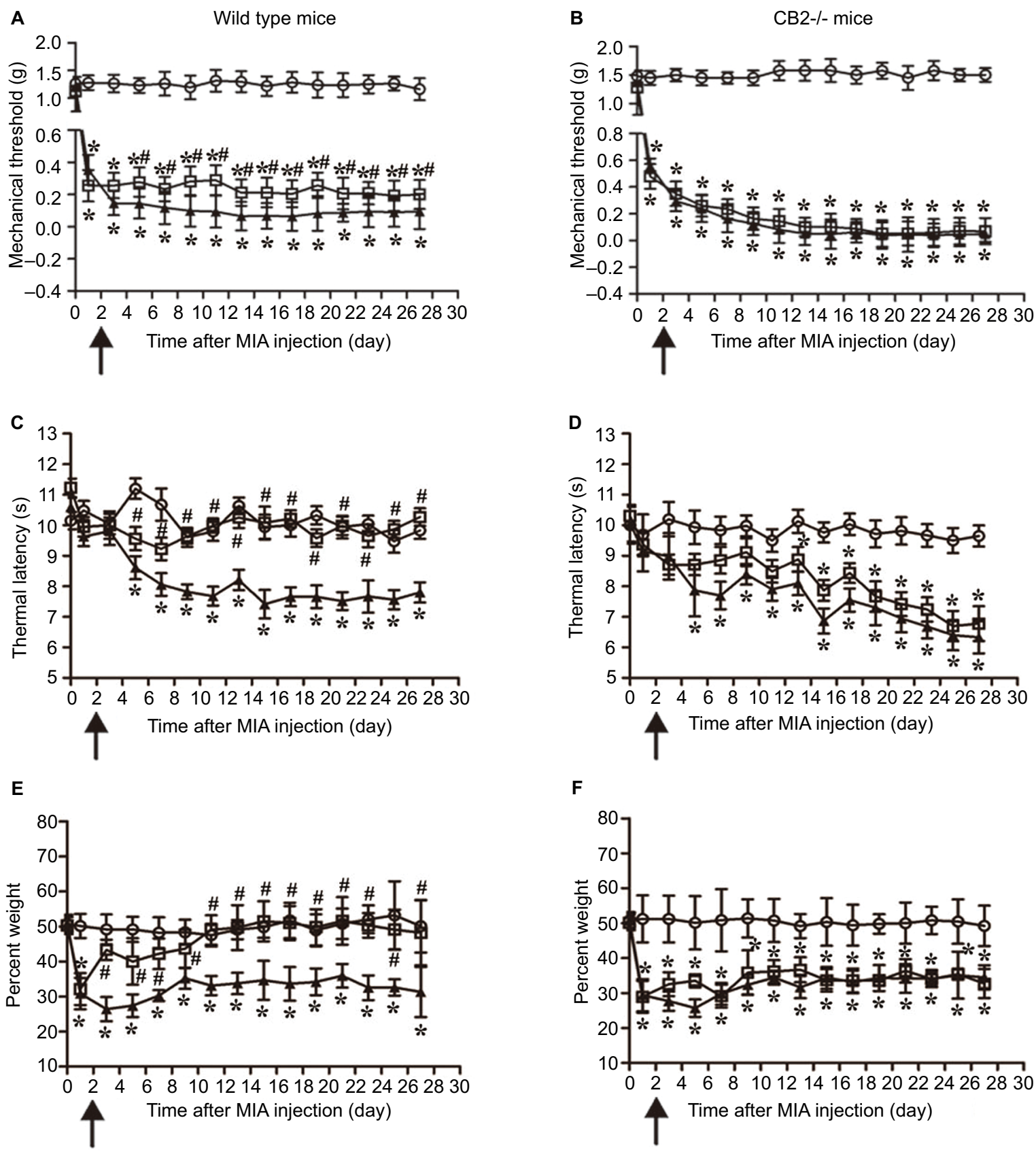

$\mathbf{F}$

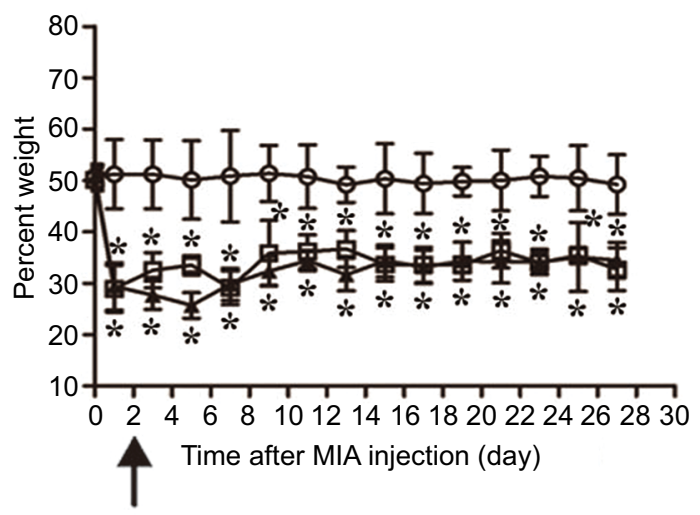

$\rightarrow \operatorname{CON}$

$\mp$ KOA

๑ EA

Figure 3 Comparison of the effects of EA on pain hypersensitivity in wild-type mice and $\mathrm{CB}^{-/-}$mice subjected to $\mathrm{KOA}$ induction.

Notes: Time course of changes in the tactile threshold in the wild-type mice (A) and $\mathrm{CB2}^{---}$mice (B) after KOA induction. Time course of changes in the thermal withdrawal threshold in the wild-type mice $(\mathbf{C})$ and $\mathrm{CB2}^{-/-}$mice $(\mathbf{D})$ after KOA induction. Effects of KOA and EA on weight bearing of wild-type mice $(\mathbf{E})$ and $\mathrm{CB2}^{-/-}$mice $(\mathbf{F})$ subjected to KOA induction. EA was administered for 30 minutes, once every other day for 4 weeks, starting from 2 days after MIA injection, as indicated by arrows. Data are expressed as mean $\pm S D$ ( $n=8$ mice in each group). ${ }^{*} P<0.05$, compared with the control group; ${ }^{*} P<0.05$, compared with the KOA group.

Abbreviations: CON, control; EA, electroacupuncture; KOA, knee osteoarthritis; MIA, monosodium iodoacetate.

\section{Discussion}

In this study, we demonstrated that EA treatment at $2 \mathrm{~Hz}+1 \mathrm{~mA}$ significantly increased the expression of CB2 receptor in fibroblast and decreased the level of IL-1 $\beta$ in the knee meniscus of KOA mice. Moreover, we also found that knockout of $\mathrm{CB} 2$ receptor can block the analgesic effect of EA and that EA had no effect on the expression of IL-1 $\beta$ in $\mathrm{CB} 2^{-/-}$mice. Our studies provided the evidence that EA can reduce the expression of IL- $1 \beta$ through activating the CB2 receptor, thus inhibiting the chronic pain in the model of KOA. 
A
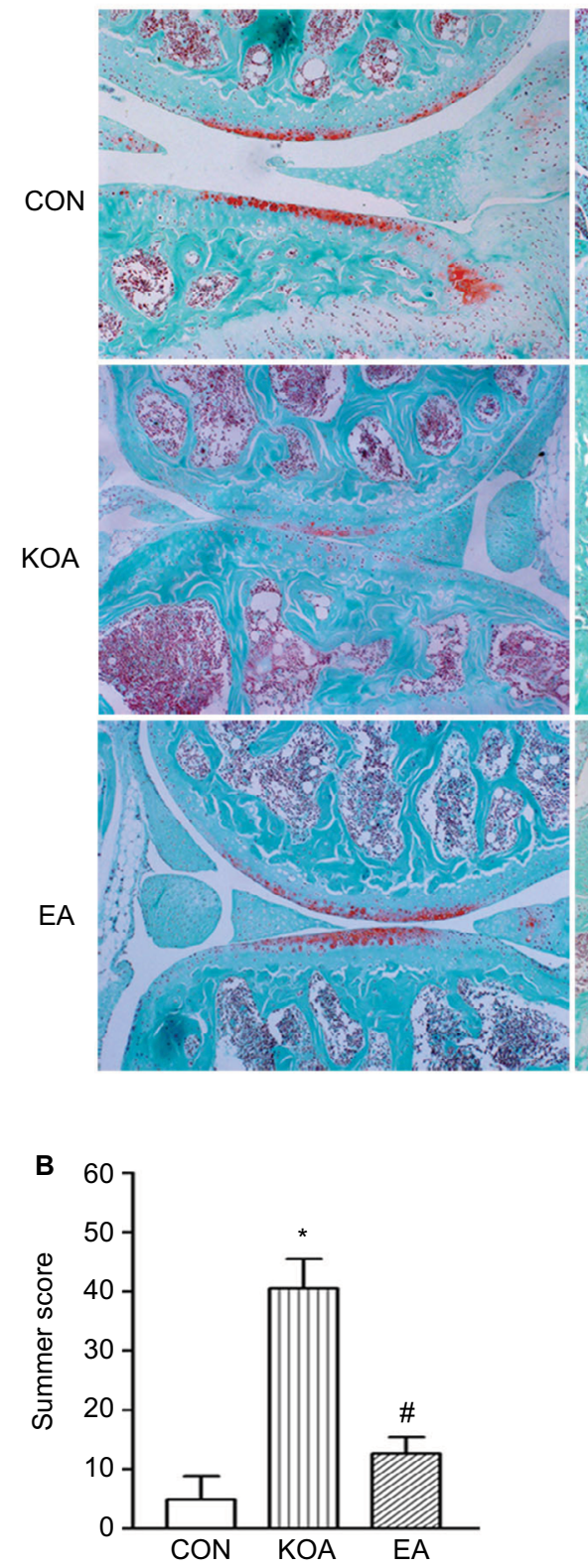

CB2-/- mice
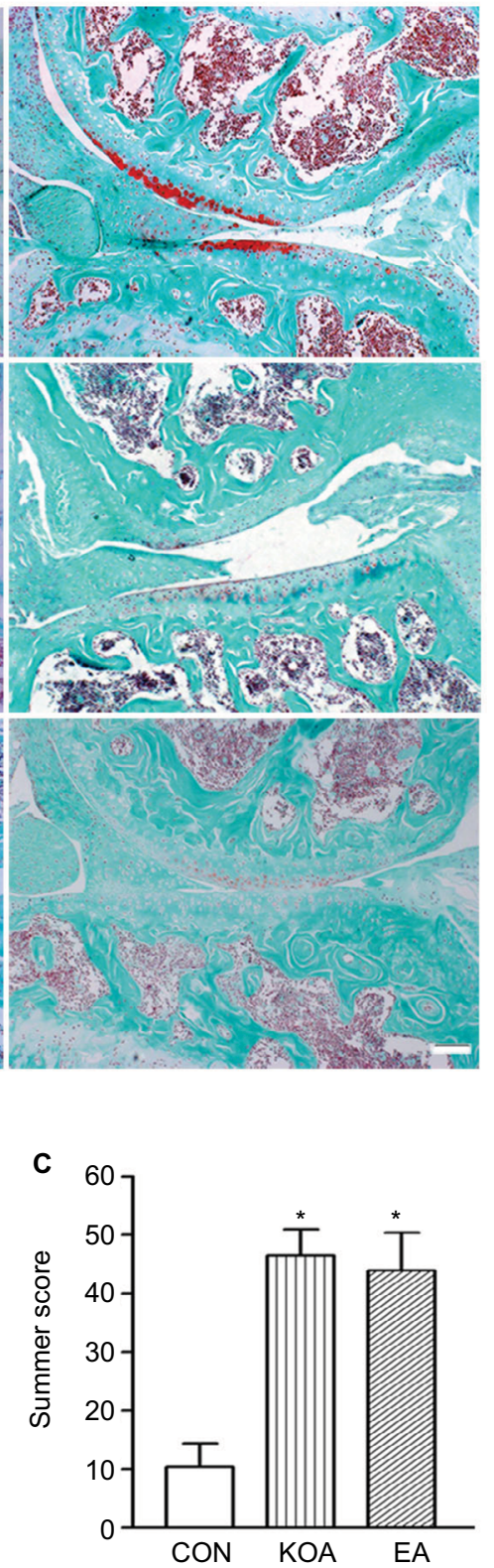

Figure 4 Comparison of the effects of EA on histological changes in wild-type mice and CB2 ${ }^{-/}$mice subjected to KOA induction.

Notes: (A) Representative histological knee joint sections (medial side) stained with Safranin O-Fast Green of wild-type mice and CB2 ${ }^{-/-}$mice. Summary data show the effect of EA on the OARSI score of wild-type mice (B) and $C B 2^{-/-}$mice (C). Data are expressed as mean $\pm S D(n=6$ mice in each group). Scale bar: I00 $\mu$ m. $* P<0.05$, compared with the control group; ${ }^{\# P}<0.05$, compared with the KOA group.

Abbreviations: CON, control; EA, electroacupuncture; KOA, knee osteoarthritis; OARSI, Osteoarthritis Research Society International.

Previous studies have demonstrated the antinociceptive effects of CB2 agonists in different models of chronic pain and arthritis. ${ }^{39-42} \mathrm{We}$ found that at $2 \mathrm{~Hz}+1 \mathrm{~mA}$, EA significantly increased tactile threshold, thermal latency, and the percentage of weight borne after KOA modeling. However, EA had no significant effects on mechanical threshold, thermal latency, and the percentage of weight borne after KOA modeling in $\mathrm{CB} 2^{-/-}$mice. These data suggest that the $\mathrm{CB} 2$ receptor participates in EA-inhibited chronic pain in KOA mice.
In addition, previous studies showed that the activated peripheral CB2 receptor can reduce inflammatory pain and neuropathic pain. ${ }^{15,16}$ In this study, we found that EA treatment at $2 \mathrm{~Hz}+1 \mathrm{~mA}$ significantly increased the expression of CB2 receptor in the knee meniscus of KOA mice.

Although the $\mathrm{CB} 2$ receptor is mainly distributed in immune cells, evidence exists that the CB2 receptor is expressed in fibroblasts, chondrocytes, and synovial cells. ${ }^{43-45}$ Consistently, our data illustrate that the CB2 receptor 


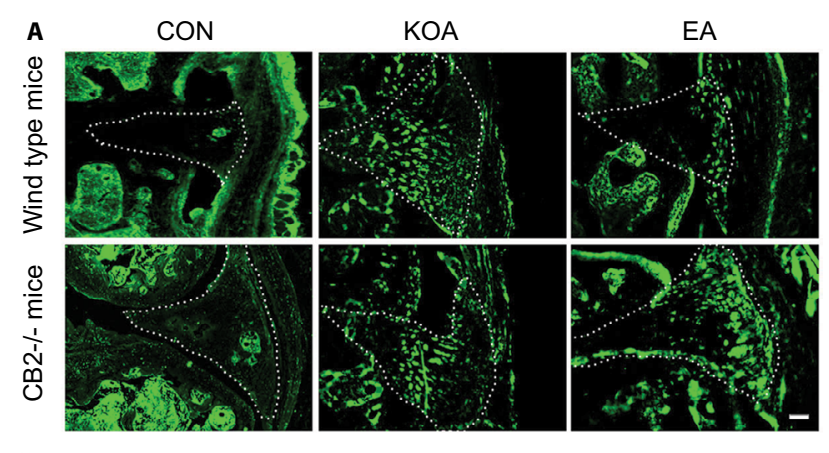

B
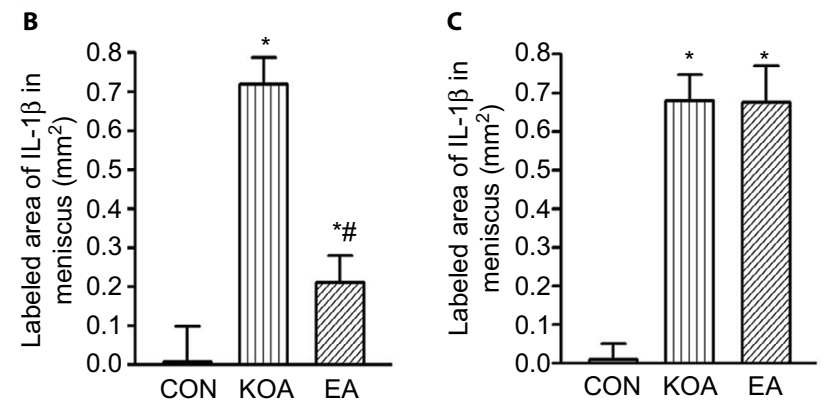

Figure 5 Quantitative analysis of the level of IL- I $\beta$ in wild-type mice and CB2mice subjected to KOA induction.

Notes: (A) Representative images showing IL-I $\beta$ of the wild-type mice and CB2 ${ }^{-1-}$ mice after KOA induction. Scale bar: $100 \mu \mathrm{m}$. Dotted line marks the menisci. Summary data show the area of IL-I $\beta$ in the menisci of the wild-type mice (B) and $\mathrm{CB}^{-1-}$ mice $(\mathbf{C})$. Data are expressed as mean $\pm \mathrm{SD}$ ( $\mathrm{n}=6$ mice in each group). $* P<0.05$, compared with the control group; ${ }^{\# P}<0.05$, compared with the KOA group.

Abbreviations: $\mathrm{CON}$, control; EA, electroacupuncture; KOA, knee osteoarthritis.

coexisted with fibroblasts in menisci and EA significantly increased the expression of $\mathrm{CB} 2$ receptor in fibroblasts. Oversecretion of pro-inflammatory cytokines, including TNF-a, IL-6, and IL-1 $\beta$, contributes to the severity and the progression of osteoarthritis. ${ }^{20}$ In addition, in vitro studies have shown that cannabinoids reduce cytokine production from rheumatoid arthritis fibroblasts. ${ }^{46} \mathrm{We}$ found that EA significantly decreased the level of IL- $1 \beta$ in the knee meniscus of KOA mice. However, EA had no effect on the level of IL-1 $\beta$ in the knee meniscus after CB2 receptor knockout. These data suggest that EA activated $\mathrm{CB} 2$ receptor in fibroblasts and then inhibited the level of IL-1 $\beta$ in the knee meniscus.

Previous studies showed that intra-articular injection of the metabolic inhibitor MIA into the knee joint caused histological changes in the cartilage. ${ }^{26,47}$ Indeed, in our experimental conditions, the MIA dose used produced a clear loss of proteoglycan and chondrocyte degeneration in the cartilage. We also found that EA significantly improved the cartilage degradation. However, EA showed no significant effect on histological changes in the cartilage after $\mathrm{CB} 2$ receptor knockout. Furthermore, cannabinoid receptor agonists have been found to protect against collagen-induced arthritis ${ }^{48}$ and to inhibit IL-1-induced cartilage degradation in vitro. ${ }^{49}$ Thus, these findings suggest that EA improved the cartilage degradation by activating the CB2 receptor and inhibiting the level of IL-1 $\beta$.

\section{Conclusion}

EA inhibited the expression of IL-1 $\beta$ through activating the $\mathrm{CB} 2$ receptor, thus inhibiting chronic pain in the model of KOA. Our findings provide novel information about the mechanisms through which EA and $\mathrm{CB} 2$ receptor activation reduce chronic pain in the KOA mouse model.

\section{Acknowledgments}

We thank Dr Nancy E Buckley for providing $\mathrm{CB} 2^{-/-}$mice. This study was supported by two grants from the National Natural Science Foundation of China (No 81473768 and No 81473488 ) and by the major project of the National Natural Science Foundation of Hubei province (No 2015CFA094) and Huanghe Talents Plan of Wuhan City in 2015.

\section{Author contributions}

$\mathrm{ML}$ and QW conceived and designed the experiments. X-CY did most of the experiments and analyzed the data; $\mathrm{C}-\mathrm{HW}$, WS, and H-PL helped with the behavior test experiments. HZ and $\mathrm{H}-\mathrm{CX}$ helped with double-immunofluorescence labeling experiments. L-XL and FG analyzed the data. CFH and JC helped to feed the animals. X-CY, J-JL and ML wrote the manuscript. All authors contributed to data analysis, drafting or revising the article, gave final approval of the version to be published, and agree to be accountable for all aspects of the work.

\section{Disclosure}

The authors report no conflicts of interest in this work.

\section{References}

1. Marchand F, Perretti M, McMahon SB. Role of the immune system in chronic pain. Nat Rev Neurosci. 2005;6(7):521-532.

2. Zhang R, Lao L, Ren K, Berman BM. Mechanisms of acupuncture-electroacupuncture on persistent pain. Anesthesiology. 2014;120(2):482-503.

3. Weiner DK, Moore CG, Morone NE, Lee ES, Kent Kwoh C. Efficacy of periosteal stimulation for chronic pain associated with advanced knee osteoarthritis: a randomized, controlled clinical trial. Clin Ther. 2013;35(11):1703-1720.e5.

4. Zogopoulos P, Vasileiou I, Patsouris E, Theocharis SE. The role of endocannabinoids in pain modulation. Fundam Clin Pharmacol. 2013;27(1): 64-80.

5. Rice AS, Farquhar-Smith WP, Nagy I. Endocannabinoids and pain: spinal and peripheral analgesia in inflammation and neuropathy. Prostaglandins Leukot Essent Fatty Acids. 2002;66(2-3):243-256.

6. Alkaitis MS, Solorzano C, Landry RP, Piomelli D, Deleo JA, RomeroSandoval EA. Evidence for a role of endocannabinoids, astrocytes and p38 phosphorylation in the resolution of postoperative pain. PLoS One. 2010;5(5):e10891. 
7. Onaivi ES, Ishiguro H, Gong JP, et al. Discovery of the presence and functional expression of cannabinoid CB2 receptors in brain. Ann NY Acad Sci. 2006;1074:514-536.

8. Wotherspoon G, Fox A, McIntyre P, Colley S, Bevan S, Winter J. Peripheral nerve injury induces cannabinoid receptor 2 protein expression in rat sensory neurons. Neuroscience. 2005;135(1):235-245.

9. Schatz AR, Lee M, Condie RB, Pulaski JT, Kaminski NE. Cannabinoid receptors $\mathrm{CB} 1$ and $\mathrm{CB} 2$ : a characterization of expression and adenylate cyclase modulation within the immune system. Toxicol Appl Pharmacol. 1997;142(2):278-287.

10. Chen L, Zhang J, Li F, et al. Endogenous anandamide and cannabinoid receptor-2 contribute to electroacupuncture analgesia in rats. $J$ Pain. 2009;10(7):732-739.

11. Su TF, Zhao YQ, Zhang LH, et al. Electroacupuncture reduces the expression of proinflammatory cytokines in inflamed skin tissues through activation of cannabinoid CB2 receptors. Eur J Pain. 2012;16(5):624-635.

12. Su TF, Zhang LH, Peng M, et al. Cannabinoid CB2 receptors contribute to upregulation of $\beta$-endorphin in inflamed skin tissues by electroacupuncture. Mol Pain. 2011;7:98.

13. Gao F, Xiang HC, Li HP, et al. Electroacupuncture inhibits NLRP3 inflammasome activation through $\mathrm{CB} 2$ receptors in inflammatory pain. Brain Behav Immun. 2018;67:91-100.

14. Zhang J, Chen L, Su T, et al. Electroacupuncture increases CB2 receptor expression on keratinocytes and infiltrating inflammatory cells in inflamed skin tissues of rats. J Pain. 2010;11(12):1250-1258.

15. Guindon J, Hohmann AG. Cannabinoid CB2 receptors: a therapeutic target for the treatment of inflammatory and neuropathic pain. $\mathrm{Br} \mathrm{J}$ Pharmacol. 2008;153(2):319-334.

16. Anand P, Whiteside G, Fowler CJ, Hohmann AG. Targeting CB2 receptors and the endocannabinoid system for the treatment of pain. Brain Res Rev. 2009;60(1):255-266.

17. Ferreira-Gomes J, Adães S, Castro-Lopes JM. Assessment of movementevoked pain in osteoarthritis by the knee-bend and CatWalk tests: a clinically relevant study. J Pain. 2008;9(10):945-954.

18. Burston JJ, Mapp PI, Sarmad S, et al. Robust anti-nociceptive effects of monoacylglycerol lipase inhibition in a model of osteoarthritis pain. Br J Pharmacol. 2016;173(21):3134-3144.

19. La Porta C, Bura SA, Aracil-Fernández A, Manzanares J, Maldonado R. Role of CB1 and CB2 cannabinoid receptors in the development of joint pain induced by monosodium iodoacetate. Pain. 2013;154(1): $160-174$.

20. Karsdal MA, Bay-Jensen AC, Lories RJ, et al. The coupling of bone and cartilage turnover in osteoarthritis: opportunities for bone antiresorptives and anabolics as potential treatments? Ann Rheum Dis. 2014;73(2): 336-348.

21. Yang S, Dubé CE, Eaton CB, McAlindon TE, Lapane KL. Longitudinal use of complementary and alternative medicine among older adults with radiographic knee osteoarthritis. Clin Ther. 2013;35(11): 1690-1702.

22. Sophocleous A, Börjesson AE, Salter DM, Ralston SH. The type 2 cannabinoid receptor regulates susceptibility to osteoarthritis in mice. Osteoarthritis Cartilage. 2015;23(9):1586-1594.

23. Zimmermann M. Ethical guidelines for investigations of experimental pain in conscious animals. Pain. 1983;16(2):109-110.

24. Buckley NE, McCoy KL, Mezey E, et al. Immunomodulation by cannabinoids is absent in mice deficient for the cannabinoid $\mathrm{CB}(2)$ receptor. Eur J Pharmacol. 2000;396(2-3):141-149.

25. Yuan XC, Zhu B, Jing XH, et al. Electroacupuncture Potentiates Cannabinoid Receptor-Mediated Descending Inhibitory Control in a Mouse Model of Knee Osteoarthritis. Front Mol Neurosci. 2018;11:112.

26. van Osch GJ, van der Kraan PM, van den Berg WB. Site-specific cartilage changes in murine degenerative knee joint disease induced by iodoacetate and collagenase. J Orthop Res. 1994;12(2):168-175.
27. Harvey VL, Dickenson AH. Behavioural and electrophysiological characterisation of experimentally induced osteoarthritis and neuropathy in C57B1/6 mice. Mol Pain. 2009;5:18.

28. Ng MM, Leung MC, Poon DM. The effects of electro-acupuncture and transcutaneous electrical nerve stimulation on patients with painful osteoarthritic knees: a randomized controlled trial with follow-up evaluation. J Altern Complement Med. 2003;9(5):641-649.

29. Selfe TK, Taylor AG. Acupuncture and osteoarthritis of the knee: a review of randomized, controlled trials. Fam Community Health. 2008;31(3):247-254.

30. Fernihough J, Gentry C, Malcangio M, et al. Pain related behaviour in two models of osteoarthritis in the rat knee. Pain. 2004;112(1-2): $83-93$.

31. Ferreira J, Santos AR, Calixto JB. The role of systemic, spinal and supraspinal L-arginine-nitric oxide-cGMP pathway in thermal hyperalgesia caused by intrathecal injection of glutamate in mice. Neuropharmacology. 1999;38(6):835-842.

32. Pigatto GR, Coelho IS, Aquino RS, Bauermann LF, Santos ARS. LightEmitting Diode Phototherapy Reduces Nocifensive Behavior Induced by Thermal and Chemical Noxious Stimuli in Mice: Evidence for the Involvement of Capsaicin-Sensitive Central Afferent Fibers. Mol Neurobiol. 2017;54(5):3205-3218.

33. Chaplan SR, Bach FW, Pogrel JW, Chung JM, Yaksh TL. Quantitative assessment of tactile allodynia in the rat paw. J Neurosci Methods. 1994;53(1):55-63.

34. Li A, Zhang Y, Lao L, et al. Serotonin Receptor $2 \mathrm{~A} / \mathrm{C}$ Is Involved in Electroacupuncture Inhibition of Pain in an Osteoarthritis Rat Model. Evid Based Complement Alternat Med. 2011;2011:6.

35. Glasson SS, Chambers MG, Van Den Berg WB, Little CB. The OARSI histopathology initiative - recommendations for histological assessments of osteoarthritis in the mouse. Osteoarthritis Cartilage. 2010;18(Suppl 3): S17-S23.

36. Richardson D, Pearson RG, Kurian N, et al. Characterisation of the cannabinoid receptor system in synovial tissue and fluid in patients with osteoarthritis and rheumatoid arthritis. Arthritis Res Ther. 2008;10(2):R43.

37. Fukuda S, Kohsaka H, Takayasu A, et al. Cannabinoid receptor 2 as a potential therapeutic target in rheumatoid arthritis. BMC Musculoskelet Disord. 2014;15:275.

38. Landis JR, Koch GG. The measurement of observer agreement for categorical data. Biometrics. 1977;33(1):159-174.

39. Yao BB, Hsieh GC, Frost JM, et al. In vitro and in vivo characterization of A-796260: a selective cannabinoid CB2 receptor agonist exhibiting analgesic activity in rodent pain models. Br J Pharmacol. 2008;153(2):390-401.

40. Whiteside GT, Gottshall SL, Boulet JM, et al. A role for cannabinoid receptors, but not endogenous opioids, in the antinociceptive activity of the CB2selective agonist, GW405833. Eur J Pharmacol. 2005;528(1-3):65-72.

41. Sain NM, Liang A, Kane SA, Urban MO. Antinociceptive effects of the non-selective cannabinoid receptor agonist CP 55,940 are absent in CB1(-/-) and not CB2(-/-) mice in models of acute and persistent pain. Neuropharmacology. 2009;57(3):235-241.

42. Cox ML, Haller VL, Welch SP. The antinociceptive effect of Delta9tetrahydrocannabinol in the arthritic rat involves the $\mathrm{CB}(2)$ cannabinoid receptor. Eur J Pharmacol. 2007;570(1-3):50-56.

43. Fede C, Albertin G, Petrelli L, et al. Expression of the endocannabinoid receptors in human fascial tissue. Eur J Histochem. 2016;60(2):2643.

44. McPartland JM. Expression of the endocannabinoid system in fibroblasts and myofascial tissues. J Bodyw Mov Ther. 2008;12(2):169-182.

45. Lowin T, Pongratz G, Straub RH. The synthetic cannabinoid WIN55,212-2 mesylate decreases the production of inflammatory mediators in rheumatoid arthritis synovial fibroblasts by activating CB2, TRPV1, TRPA1 and yet unidentified receptor targets. J Inflamm (Lond). 2016;13:15.

46. Selvi E, Lorenzini S, Garcia-Gonzalez E, et al. Inhibitory effect of synthetic cannabinoids on cytokine production in rheumatoid fibroblastlike synoviocytes. Clin Exp Rheumatol. 2008;26(4):574-581. 
47. van der Kraan PM, Vitters EL, van de Putte LB, van den Berg WB. Development of osteoarthritic lesions in mice by "metabolic" and "mechanical" alterations in the knee joints. Am J Pathol. 1989;135(6):1001-1014.

48. Sumariwalla PF, Gallily R, Tchilibon S, Fride E, Mechoulam R, Feldmann M. A novel synthetic, nonpsychoactive cannabinoid acid (HU-320) with antiinflammatory properties in murine collagen-induced arthritis. Arthritis Rheum. 2004;50(3):985-998.
49. Mbvundula EC, Bunning RA, Rainsford KD. Arthritis and cannabinoids: HU-210 and Win-55,212-2 prevent IL-1alpha-induced matrix degradation in bovine articular chondrocytes in-vitro. J Pharm Pharmacol. 2006;58(3):351-358. 


\section{Supplementary material}

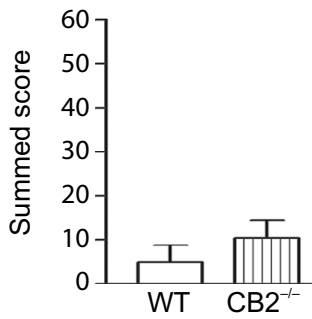

Figure SI Comparison of the OARSI score in WT mice and $\mathrm{CB}^{-/-}$mice after saline injection.

Notes: Summary data show the OARSI score of WT mice and $C B 2^{-/-}$mice after saline injection. Data are expressed as mean $\pm S D$ ( $n=6$ mice in each group).

Abbreviations: OARSI, Osteoarthritis Research Society International; WT, wildtype.

The Journal of Pain Research is an international, peer reviewed, open access, online journal that welcomes laboratory and clinical findings in the fields of pain research and the prevention and management of pain. Original research, reviews, symposium reports, hypothesis formation and commentaries are all considered for publication.
The manuscript management system is completely online and includes a very quick and fair peer-review system, which is all easy to use. Visit http://www.dovepress.com/testimonials.php to read real quotes from published authors. 